\title{
Mauricio Golfarini
}

\section{RESUMEN}

La Teoría del Acontecimiento Didáctico (TAcDi) toma elementos de la transposición didáctica de Chevallard, específicamente su noción de cronogénesis, y los reinterpreta a partir de la concepción de temporalidad del psicoanálisis lacaniano. De tal manera, la naturaleza compleja del tiempo lógico permite encontrar aspectos poco explorados en el despliegue temporal del fenómeno didáctico, como el papel de las escansiones o mociones suspendidas. En el presente trabajo se revisa algunos aspectos importantes del tiempo lógico en la TAcDi, a la vez que se plantea un modo adicional de concebir los estadios escansivos en su relación con el saber (supuesto saber) del enseñante y el nosaber del enseñado dentro de la ficción didáctica.

\section{PALABRAS CLAVE}

Transposición; Didáctica; Temporalidad; Escansión; Acontecimiento; Psicoanálisis

\section{TO KNOW THAT THE OTHER KNOWS, TO KNOW WHAT THE OTHER KNOWS: NOTE ON THE STRUCTURE OF THE LOGICAL TIME IN LACAN AND THE DIDACTIC EVENT}

\begin{abstract}
The Theory of Didactic Event takes elements from the didactic transposition of Chevallard, specifically its notion of chronogenesis, and re-interprets it from the conception of temporality of the lacanian psychoanalysis. In such way, the complex nature of the logical time allows to find unexplored aspects in the temporary unfolding of the didactic phenomenon like the role of the scansions or suspended motions. The present work reviews some important aspects of the logical time in the Theory of the Didactic Event, and simultaneously considers an additional way to conceive the scansive stages in their relation with the knowledge of the teacher and non-knowledge of the taught one within the didactic fiction.
\end{abstract}

\section{KEYWORDS}

Transposition; Didactics; Temporality; Scansion; Event; Psychoanalysis 


\section{BREVE HISTORIA DEL TIEMPO LÓGICO}

En 1945, tras un mutismo intelectual que duró tanto como la Segunda Guerra Mundial, Jacques Lacan aceptó la invitación de Christian Zervos, director de Les Cahiers D’Art, para colaborar en un número especial de la revista francesa que buscaba llenar el vacío del período de la ocupación alemana. Es así como apareció “El tiempo lógico y la aserción de certidumbre anticipada” junto a una exótica combinación de ensayos, reproducciones de pinturas, poesía, etc. Pero las ideas que aparecen en el complejo texto del psicoanalista tienen un nacimiento bastante anterior. En 1935 Lacan había escuchado un acertijo, un rompecabezas lógico presentado por el matemático André Weiss, posiblemente como un simple entretenimiento de sobremesa, pero que lo mantuvo ocupado por un largo tiempo desde que “[...] el ingenioso anfitrión de una noche lo trajo a nuestra reflexión” (LACAN, 1987a, p.189). Elizabeth Roudinesco comenta que en su afán por resolver el rompecabezas "Lacan no había podido conciliar el sueño. A las tres de la mañana, había telefoneado a Weiss que le había dado la tan deseada respuesta. Weiss, sin embargo, estaba furioso de que lo hubieran despertado a la mitad de la noche” (ROUDINESCO, 1994, p.263).

Resumidamente, el acertijo que había provocado el insomnio de Lacan sitúa a tres convictos ante una maquiavélica prueba ideada por un carcelero. A los tres se les coloca un círculo en sus respectivas espaldas, de los que en total hay tres blancos y dos negros, y luego se les permite mirarse entre sí de manera que cada cual puede ver el color que portan los otros pero les es imposible saber cual es el color del círculo que cargan ellos mismos. Les está totalmente prohibido comunicarse (oralmente) y el primero que consiga averiguar el color de su círculo y lo pueda argumentar lógicamente será liberado. A continuación se les fija a cada uno un círculo blanco y acondicionados de ésta manera, tras algunos avances y detenciones (mociones suspendidas), los tres presos no tardan mucho tiempo en presentarse simultáneamente al carcelero aduciendo:

Dado que mis compañeros eran blancos, pensé que, si yo fuese negro, cada uno de ellos hubiera podido inferir de ello lo siguiente: «Si yo también fuese negro, el otro, puesto que debería reconocer en esto inmediatamente que él es blanco, habría salido en seguida; por lo tanto yo no soy un negro». Y los dos habrían salido juntos, convencidos de ser blancos. Si no hacían tal cosa, es que yo era un blanco como ellos. Así que me vine a la puerta para dar a conocer mi conclusión (LACAN, 1987a, p.188). 
Para Lacan la solución al acertijo, explicada por Weiss, es perfecta, demasiado perfecta para ser un simple truco (para la lógica clásica ésta solución es errónea), por lo que asume el problema como un sofisma o "un ejemplo significativo para resolver las formas de una función lógica en el momento histórico en que su problema se presenta al examen filosófico” (LACAN, 1987a, p.189). Lacan se toma una década para elaborar su propia solución, presentando al final una visión radicalmente distinta del despliegue de los razonamientos de los actores implicados en lo que se conoce como tiempo lógico, y que en muchos aspectos se adelanta a los hallazgos de Kripke (1959) en el terreno de la lógica modal, y de MacCarthy (1990) en el de los puzzles lógicos. Lo revolucionario en la solución lacaniana es que "sólo la virtud lógica conduce al hombre a la verdad, es decir, a la aceptación del otro según una dialéctica del reconocimiento y del desconocimiento” (ROUDINESCO, 1994, p.265), introduciendo una nueva forma de explicar la intersubjetividad en términos horizontales, a diferencia de lo pobremente establecido por Freud.

\section{EL TIEMPO LÓGICO EN LA TEORÍA DEL ACONTECIMIENTO DIDÁCTICO}

La Teoría del Acontecimiento Didáctico es el marco teórico aplicado por el Departamento de Psicología de la Educación y Didáctica de la Facultad de Humanidades y Ciencias de la Educación (Universidad de la República, Montevideo, Uruguay) en su línea de investigación "Estudio de lo didáctico como acontecimiento discursivo e intersubjetividad” (EDADI). Como explica Behares (2006, p.234), esta teoría estudia el fenómeno didáctico mediante un "esfuerzo heurístico [que] nos lleva a circunscribirlo a partir de la teoría de la transposición didáctica y a interpretarlo en el marco de la teoría marxista del discurso y el psicoanálisis lacaniano”. Esta visión psicoanalítica específica permite correlacionar fácilmente las nociones de cronogénesis, heredada de la transposición didáctica de Chevallard (1991), con el tiempo lógico de Lacan. Según A. M. Fernández, participante de la línea de investigación mencionada, la conexión entre las dos nociones surge en el momento que “[...] Chevallard hace uso, en forma explícita del concepto freudiano de posterioridad y, en forma implícita, de la concepción temporal desarrollada por Lacan” (FERNÁNDEZ, 2005, p.111).

El tiempo lógico posee una estructura triádica: el instante de la mirada, el tiempo para comprender y el momento de concluir. El primer elemento de la tríada lo forman 
aquellos razonamientos que un sujeto puede realizar a partir de lo que se le presenta a simple vista, de lo que los sentidos le aportan directamente. Es un proceso casi instantáneo, tan veloz que aparenta ser la respuesta natural a una configuración o estado de cosas. En los términos del sofisma sería lo que le tomaría a uno de los presos para darse cuenta que su círculo es blanco si viera a los otros dos cargando círculos negros, y bajo la forma de acontecimiento didáctico, es el tiempo que al enseñado le toma para darse cuenta que el saber que el enseñante le presenta no es algo que reconozca, que es algo nuevo que contradice lo viejo, algo que no-sabe. En cambio el tiempo para comprender es el tiempo en que el sujeto busca determinar su posición frente al otro (pequeño otro de Lacan) mediante una serie de pensamientos mas o menos complejos, en los que está implicado el ponerse en el lugar del otro para anticipar su accionar. Desde el acontecimiento didáctico, se puede ver esta actitud en el enseñante cada vez que anticipa las deducciones y respuestas de los enseñados frente al saber expuesto: "Hay, entonces, el tiempo de la enseñanza, caracterizado por la anticipación, y el tiempo del aprendizaje de naturaleza retroactiva” (BEHARES, 2004, p.56).

Por último está el momento de concluir, en el que el sujeto se apresura a asumir su estado frente a los demás, a designarse a si mismo dueño de cierto conocimiento por temor a que los otros revelen algo que invalide todo el esfuerzo invertido durante el tiempo para comprender, y que en el acontecimiento didáctico se puede traducir como la urgencia por adueñarse del saber, así sea muy precariamente, de forma de posicionarse como aquel que sabe lo que sabe el otro (enseñado) o aquel que sabe qué sabe el otro (enseñante). Este componente de la tríada, común a las dos posiciones de los sujetos del acontecimiento, es el que funciona como catalizador del despliegue temporal, ya que permite desencadenar nuevas instancias que serían imposibles si no ocurriera cierta forma de "cierre" momentáneo de los procesos previos. Sin el momento del concluir los sujetos permanecerían cristalizados en sus respectivas posiciones anticipatorias o retroactivas respecto a determinado saber específico, sin poder avanzar a otro.

\section{ESCANSIONES Y EPICICLOS}

Como lo describe Lacan en la explicación de su sofisma, la evolución del proceso no es lineal sino que implica una cantidad variable de mociones suspendidas o escansiones, dependiente del número de individuos involucrados, en las que se plantea un retorno al 
instante de la mirada - tiempo de comprender - momento de concluir. Es como si todo empezara de nuevo a partir de una duda que surge luego de iniciado el movimiento hacia a la salida, pero sin que ello implique que todo lo razonado hasta el momento quede invalidado: “[...] algún progreso lógico tiene que haberse cumplido” (LACAN, 1987a, p.191). Una manera de representar gráficamente tales mociones suspendidas es hacer un paralelismo con las órbitas planetarias de la cosmología ptolemaica. En dicho modelo del universo, los planetas, en su interminable recorrido por las esferas celestes, circunstancialmente se desplazaban en sentido opuesto al normal, exhibiendo un "extraño movimiento errante" (SAGAN, 1998, p.51). Del mismo modo, en el acontecimiento didáctico es posible ver esos epiciclos en la evolución temporal del proceso: un conflicto entre saber viejo - saber nuevo según Chevallard que se puede correlacionar con la “posición de angustia y la posición de deseo respecto al saber” (BLEZIO, 2005, p.63), la actualización de la estructura (inconsciente) en la que "irrumpe o se manifiesta el Real tras la falta de los sujetos” (BEHARES, 2006, p.236) (avance), una nueva inestabilidad (retroceso), una nueva actualización (avance)... y así sucesivamente, sin que esto signifique retornar al mismo punto inicial (nada se enseña y nada se aprende) ni tampoco llegar a un final absoluto (todo se enseña y todo se aprende). Tal como en esa vieja cosmología, la escasa notoriedad de los epiciclos induce la impresión de un movimiento continuo, perfecto, sin sobresaltos, asimilable a alguna forma de acumulación del conocimiento desde otras posiciones teóricas, pero que desde la teoría del acontecimiento didáctico se revela como una característica más de la ficción didáctica: "Hay dos regímenes del saber, articulados en sincronía para permitir el tiempo didáctico legal. El tiempo de aprendizaje no es simplemente acumulativo, progresivo” (FERNÁNDEZ, 2005, p.111).

\section{ARTEFACTOS EGOCÉNTRICOS}

El sofisma de Lacan pertenece a una categoría de problemas lógicos que han sido intensamente estudiados desde las ciencias computacionales y las ciencias cognitivas. Por ejemplo, la versión conocida como el puzzle de los “niños embarrados” (muddy children) ha sido objeto de análisis mediante estructuras de Kripke (FAGIN et al, 1995), un tipo de herramienta de la lógica modal que permite revelar la secuencia de deducciones que hace cada actor en la situación que se plantea. El mismo recurso se puede utilizar para abordar el 
sofisma lacaniano ${ }^{1}$, poniendo de manifiesto la validez lógica de la solución inicialmente propuesta por Weiss y el esfuerzo notable de Lacan por formalizarla introduciendo las mociones suspendidas.

Adicionalmente, mediante tales procedimientos se puede argumentar que los estadios escansivos, en los que los sujetos hacen una revisión de sus deducciones previas, son efecto de un discurso realizado siempre en primera persona, lo que limita la posibilidad de pensar desde el lugar de los “otros”, generando posteriormente una serie de dudas sobre la validez de los razonamientos ya efectuados. De tal manera se puede sostener que las escansiones son una forma de artefacto egocéntrico, o sea, algo que no se puede justificar lógicamente sino que surge por el efecto de un discurso permanentemente centrado en el “yo”. Como ejemplo de tal afirmación basta estudiar cómo se modifica la dinámica del sofisma cuando las respuestas de los presos se cambian de "si yo cargara un círculo negro...” a "si alguno de nosotros cargara un círculo negro...”.

Trasladando esto último al contexto del acontecimiento didáctico es fácil darse cuenta de dónde surge la duda que desencadena el proceso escansivo. El mismo artefacto egocéntrico se manifiesta, luego de la interacción con un nuevo saber, como la posibilidad inicial de afirmar que “ahora yo sé qué es lo que saben mis alumnos” (enseñante) y "ahora yo sé eso que el maestro sabe” (enseñado), seguido por una incertidumbre sobre tales afirmaciones al notar que el “otro” no necesariamente piensa igual que “yo”, que el esquema de sus razonamientos puede ser diferente. Por un momento parece ser inevitable repetir todo el proceso desde el principio, se inicia una forma de revisión mental de lo realizado hasta que se alcanza nuevamente la certeza sobre "lo que sabe el otro" facilitada, la mayor parte de las veces, por indicios no verbales como la mirada, la expresión de los rostros, el "lenguaje corporal”, etc. En otros casos la duda puede quedar explicitada y el proceso escansivo se trasmuta en la práctica conocida como “repaso”, donde se reproduce, con pocos cambios, el mismo esquema aplicado previamente.

Por último, el paralelismo con el viejo "sistema del mundo" de Ptolomeo manejado antes sigue siendo útil si se considera que la existencia de los epiciclos sólo se podía justificar en un universo geocéntrico, eran artefactos geocéntricos, cosa que la cosmología

\footnotetext{
${ }^{1}$ El estudio del sofisma de Lacan mediante estructuras de Kripke y la semántica de mundos posibles forma parte de un trabajo aún no publicado que el autor viene desarrollando dentro de la línea de investigación del Departamento de Psicología de la Educación y Didáctica de la Facultad de Humanidades y Ciencias de la Educación (Universidad de la República - Montevideo, Uruguay).
} 
moderna corrigió al colocar al Sol como eje de las órbitas planetarias. A partir de la revolución copernicana la perfección del universo geocéntrico pasó a ser una entelequia, del mismo modo que la supuesta omnisciencia de los sujetos implicados en el hecho didáctico queda expuesta como una faceta más de una ficción bajo la luz de la Teoría del Acontecimiento Didáctico.

\section{CONCLUSION}

En el presente trabajo se han expuesto, en forma muy resumida, ciertas particularidades que surgen de la interpretación de la temporalidad y la intersubjetividad del fenómeno didáctico basada en el psicoanálisis lacaniano. Pero aún hay muchos aspectos de dicha temporalidad escasamente estudiados. La relevancia que Lacan asignó a su formulación del tiempo lógico queda patente en el famoso "Discurso de Roma” en donde, a propósito del estatuto del psicoanálisis manifiesta que éste:

No dará fundamentos científicos a su teoría como a su técnica sino formalizando de manera adecuada estas dimensiones esenciales de su experiencia que son, con la teoría histórica del símbolo: la lógica intersubjetiva y la temporalidad del sujeto (LACAN, 1987b, p.278).

A partir de tal afirmación se puede entender que una exploración mas profunda de la estructura de tales tiempos y su impacto en el contexto de la Teoría del Acontecimiento Didáctico representa un camino interesante para afianzar el estatus de ésta última dentro del las Ciencias de la Educación, permitiéndole adicionalmente, hacer uso de nuevas herramientas para abordar los fenómenos didácticos. Si la dinámica interpersonal que se manifiesta como tiempo lógico es “[...] una forma general de acercamiento y recorrido ante cualquier conocimiento (aprendizaje)” (FERNÁNDEZ, 2005, p.112), entonces cualquier avance en el esclarecimiento de tal dimensión temporal tendrá como consecuencia un aumento en el cuerpo teórico de la TAcDi y una mejor comprensión de algunas facetas del fenómeno didáctico que aún se presentan como “oscuras”. 


\section{REFERENCIAS}

BEHARES, L. Materialidades del saber en didáctica a partir del concepto de "transposición”. En: . (Dir.). Didáctica mínima: los acontecimientos del saber. Montevideo: Psicolibros - Waslala, 2004. p. 31-64.

La Enseñanza en el campo de la sordera: reflexiones desde la teoría del acontecimiento didáctico. Educação Temática Digital, Campinas, v. 7, n. 2, p. 228-243, jun. 2006.

BLEZIO, C. Intersubjetividad y resignificación. En: BEHARES, L. et al. (Comp.). Enseñanza del saber - saber de la enseñanza. Montevideo: Departamento de Publicaciones de la FHCE. 2005. p. 61-66.

CHEVALLARD, Y. La Transposición Didáctica: Del Saber Sabio al Saber Enseñado. 1. ed. Buenos Aires: Aique. 1997.

FAGIN, R. et al. Reasoning about knowledge. Cambridge: MIT Press. 1995.

FERNÁNDEZ, A. M. Un estudio sobre el lugar del saber científico y el saber enseñando en la didáctica. En: BEHARES, L. (Dir.). Didáctica mínima: los acontecimientos del saber. Montevideo: Psicolibros - Waslala, 2004. p. 85-121.

KRIPKE, S. A completeness theorem in modal logic, Journal of Symbolic Logic, n. 24, p. 1-14, 1959.

LACAN, J. El tiempo lógico y el aserto de certidumbre anticipada: un nuevo sofisma. En: . Escritos I. Buenos Aires: Siglo XXI, 1987a. p. 187-203. . Función y campo de la palabra y del lenguaje en psicoanálisis. En:

Escritos I. Buenos Aires: Siglo XXI, 1987b. p. 227-310.

MACCARTHY, J. Formalization of two puzzles involving knowledge En: LIFSCHITZ, V. (Ed.). Formalizing common sense: papers by John McCarthy. New Jersey: Ablex, 1990.

ROUDINESCO, E. Lacan: esbozo de una vida, historia de un sistema de pensamiento. 1. ed. Buenos Aires: Fondo de Cultura Económica, 1994.

SAGAN, C. Cosmos. 4. ed. Barcelona: Planeta, 1998. 


\section{DOSSIÊ \\ Cuerpo, Lenguaje y Enseñanza \\ Área Temática: Diferenças e Subjetividades em Educação}

Nota: Este trabajo forma parte del Grupo de Trabajo “Cuerpo y Enseñanza” correspondiente a la Línea de Investigación Estudio de lo didáctico como Acontecimiento Discursivo e Intersubjetividad (EDADI) del Departamento de Psicología de la Educación y Didáctica de la Facultad de Humanidades y Ciencias de la Educación, Universidad de la República, Montevideo - Uruguay. Se vincula a su vez a los acuerdos de trabajo realizados entre este Departamento y el Dpto. de Investigación del ISEF/ UdelaR.

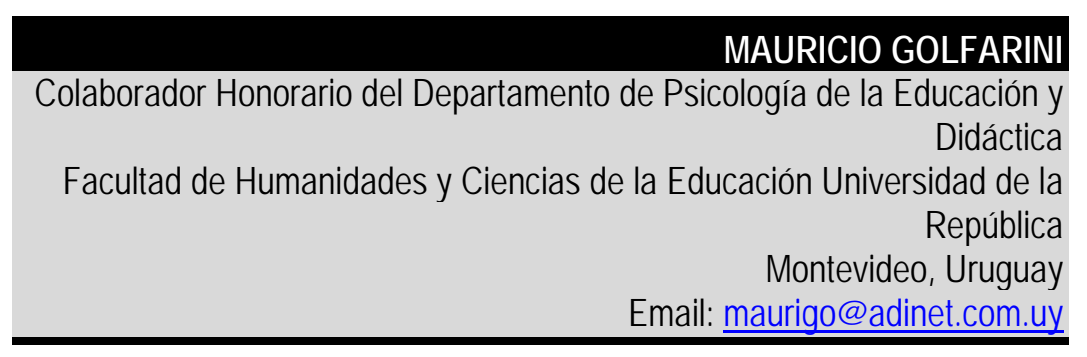

